\title{
Mammographic Registration: Proposal and Evaluation of a New Approach ${ }^{\star}$
}

\author{
Robert Martí ${ }^{1}$, David Raba ${ }^{1}$, Arnau Oliver ${ }^{1}$, and Reyer Zwiggelaar ${ }^{2}$ \\ ${ }^{1}$ Computer Vision and Robotics Group, University of Girona \\ Av. Lluís Santaló 17071, Spain \\ \{marly, draba, aoliver\}@eia.udg.es \\ http://eia.udg.es/〜marly \\ 2 Department of Computer Science, University of Wales, \\ Aberystwyth Ceredigion, SY23 3DB, Wales, UK \\ rrz@aber.ac.uk
}

\begin{abstract}
The detection of architectural distortions and abnormal structures in mammographic images can be based on the analysis of bilateral and temporal cases. This paper presents a novel method for mammographic image registration inspired by existing robust point matching approaches. This novel method is compared with other registration approaches proposed in the literature using both quantitative and qualitative evaluation based on similarity metrics and ROC analysis (ground truth provided by an expert radiologist). Initial evaluation is based on mammographic data of 64 women with malignant masses which indicates the accuracy and robustness of our method.
\end{abstract}

\section{Background}

Image registration has been widely used in medical applications for quite a while now, and the analysis of mammographic images is not an exception. An added difficulty of trying to register mammographic images is their projective nature. Nevertheless, different approaches have been adopted to obtain an alignment and minimise effects due to acquisition factors such as patient movement, breast compression and other image related factors (film exposure and energy). Most of the published approaches (including the early works of Sallam and Bowyer [1] and Karssemeijer and te Brake [2]) use breast boundary information as it is relatively easy to extract and provides important information about the breast deformation. Another group of approaches can be classified as being intensity based, where the deformation is recovered maximising a measure of similarity between images. The use of an intensity measure to recover global transformations has been reported to obtain robust results [3, but can not account for severe local distortions and additional steps are needed. In addition to the breast boundary, information about the deformation of internal regions is also necessary in order to obtain a robust registration. This has been used by different authors [4,5, 1].

\footnotetext{
* Research partiallly supported by the Juan de la Cierva Programme and research grant no. TIN2005-08792-C03-01
} 
Although non-linear registration (warping methods) of mammographic images has been regarded by some authors as non-appropriate [3, it is our belief, which is corroborated by other authors [5], that non-linear transformation can also be successfully used. However, special care has to be taken in choosing the transformation function and its parameters (in particular, regularisation factors which ensure smoothness and continuity). It is true that a naive implementation can lead to non-realistic transformations.

The method presented here is an evolution of our initial proposal 4], focusing now on providing a robust framework for establishing point correspondence between mammograms. The novelty of this paper is twofold. Firstly, we introduce and adapt different concepts of robust point matching approaches to the proposed registration approach. Secondly, an evaluation is presented comparing our method to other existing approaches in terms of similarity measures and ROC curves using a relatively large number of cases. Although initial results, this work shows that image registration can be successfully used to asses temporal changes in mammograms such as involution of breast tissue, the detection of masses or architectural distortions.

\section{Method}

The registration methodology presented here is based on robustly matching interest points in two mammographic images of the same view (either $M L O$ or $C C)$. The algorithm extracts interest points found in the boundary and the internal breast region, and applies a robust point matching approach obtaining a non-linear transformation. Registered images are used for detecting possible abnormalities in contralateral mammograms (comparing left and right breasts) by subtracting images and measuring local measures of similarity.

An initial pre-processing step segments the breast boundary and extracts interest points from the boundary and internal regions. A distinction between boundary and internal structures is made. Boundary information is used to restrict the detection area of internal structures and is also a good initial estimate of the breast deformation. In this paper, the breast boundary is obtained by simple thresholding and morphological opening operations. Subsequently, interest points are obtained from this boundary by computing their maximal local curvature. Interest points internal to the breast are also extracted using a criteria of local maximal curvature after a line detection algorithm is applied to the breast region. This pre-processing is similar to the one presented in [4].

\subsection{Point Matching Algorithm}

The idea behind the registration methodology of this paper is inspired by robust point correspondence methods proposed by various authors $[6,7,8,8$. The common approach from the cited methods is the use of an iterative process in order to minimise correspondence errors. Those errors are related to a cost matrix $\left(C_{i j}\right)$ which describes the cost of matching one point $i$ in one image (row $i$ ) with a point $j$ in the second image (column $j$ ). The elements of this matrix are 
obtained using different point error measures such as Euclidean distance, shape contexts [7, local intensity information, gradient, etc. Additionally, relaxation labelling or soft assign methods can be applied to the cost matrix in order to minimise ambiguous matchings as in 8 . Relaxation methods are not applied in this work but will be investigated in the future. The minimisation of the cost matrix yields potential point matches which are used for transforming one point set $(p)$ in order to match the other $(q)$. The transformed points $p$ and $q$ are used for building the cost matrix for the next iteration. The stopping criteria of the iterative process is usually stated in terms of a maximum number of iterations or if the number of matches does not change with respect the last iteration.

Cost matrix. The Euclidean distance between points has perhaps been the most common distance measure for point matching. This is the case of Closest Iterative Point based methods (ICP) []. Shape Contexts (SC), originally proposed by Belongie et al. [7] are rich shape descriptors based on building local point distribution histograms. Thus, at a point level $p_{i}, S C$ provide information about point distribution relative to that point $p_{i}$. A cost of matching points in both images can be obtained by comparing those local histograms. Normalised Cross-Correlation $(N C C)$ is a well known measure of similarity which has been used for many applications in computer vision. Perhaps one of the most common is template matching, obtaining the position of a known template in a larger image. $N C C$ computed within a local grey-level neighbourhood will be used as our third distance measure. The main drawback of using local similarity measures is that shape and point relationships are under-represented. For this reason, $N C C$ will be used in combination of the above measures to ensure topological point relationships. Given the set of costs $C_{i j}$, one-to-one matches are obtained minimising the total matrix cost $H(\pi)=\sum_{i} C\left(p_{i}, q_{\pi(i)}\right)$, where $\pi(i)$ denotes all permutation. This minimisation (optimal assignment problem) is obtained using the Hungarian method, as in 7 .

Transformation. Points are transformed using the matches found in the previous step. In the first iteration, an affine transformation is used in order to recover global misregistration. In subsequent iterations, the Thin-Plate Splines (TPS) is used to obtain a smooth transformation between matched points. For a set of $d$ dimensional points $x$, the Thin-Plate approximation function is defined as a sum of $d$ independent functionals $J_{m}^{d}$ minimising a measure of bending energy (related to $m$ order derivatives, $m=d=2$ is used here).

$$
J_{m}^{d}(u)=\sum_{k=1}^{d} J_{m}^{d}\left(u_{k}\right)
$$

The solution, $u(x)$, is obtained by solving a linear system of equations,

$$
u(x)=\sum_{\nu=1}^{M} a_{\nu} \phi_{n u}(x)+\sum_{i=1}^{n} w_{i} U\left(\left|\boldsymbol{p}_{\boldsymbol{i}}-x\right|\right)
$$


where $\phi_{n u}(x)$ defines the TPS behaviour away from the control points, $U$ are the thin plate basis functions and $a_{\nu}$ and $w_{i}$ are the parameters of the transformation. The smoothness of the TPS transformation can be controlled by introducing a regularisation term $(\lambda)$ in the transformation (see [9]) weakening the interpolation condition $\left(q_{i}=u\left(p_{i}\right)\right)$.

$$
J_{\lambda}(u)=\frac{1}{n} \sum_{i=1}^{n}\left|q_{i}-u\left(p_{i}\right)\right|^{2}+\lambda J_{m}^{d}\left(u_{k}\right)
$$

For $\lambda$ values close to zero, the transformation interpolates exactly for each control point (the original TPS transformation), while for larger values we obtain smoother approximating transformations. This regularisation is used in the iterative process, where larger $\lambda$ values are used in the initial iterations decreasing its value depending on an error fitness measure.

Outliers. The Hungarian method obtains optimal matches for all points in the cost matrix. For some applications, and mammographic registration is one of them, a large number of outliers is expected in both images. In this sense, the original cost matrix is enlarged with a percentage of dummy points, points to which real points will be assigned if a better match is not found. The number of dummy matches depends on two parameters defined experimentally: the cost of matching to a dummy point (which should be small enough to allow dummy matches but at the same time large enough to obtain a significant number of real matches) and also the number of dummy points allowed (as a percentage of the total number of points). Experimentally, and although exact values are not particularly critical, we have experienced that a dummy point percentage of $30-40 \%$ with a cost of 0.1 provide the best results. Moreover, not all matches are taken into account, only those with minimal cost compared to its neighbourhood are selected as final matches. This neighbourhood criteria is implemented as a graph proximity problem.

\section{Results}

\subsection{Qualitative Results}

Here we qualitatively show the results of the registration algorithm. The described point matching algorithm is applied in two different steps. Initially, breast boundary points alone are used as interest points for finding potential matches. Subsequently, a second matching process is started in order to obtain matches for the internal points. The matching in this second step is constrained by the transformation found in the boundary matching process. This constraint is applied to the cost matrix where matches for boundary points are enforced to remain constant. Fig. 1 shows the different steps of the registration.

Fig. 2 shows original images and registration results and the difference image using the proposed method (rpm) of the example matched in Fig. 1. 


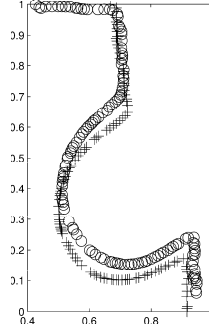

Initial

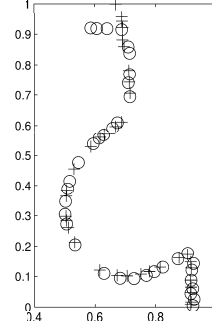

$\mathrm{BB}$ (it 1)

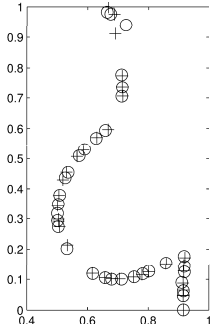

BB (it 3)

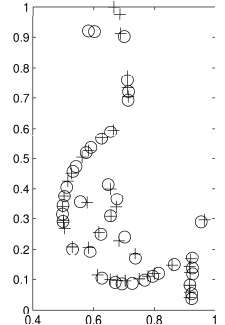

IP (it 1)

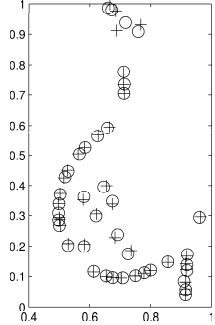

IP (it 3)

Fig. 1. Registration using breast boundary (BB) and internal points (IP) in different iterations. Crosses (circles) refer to control points from the reference (target) images.

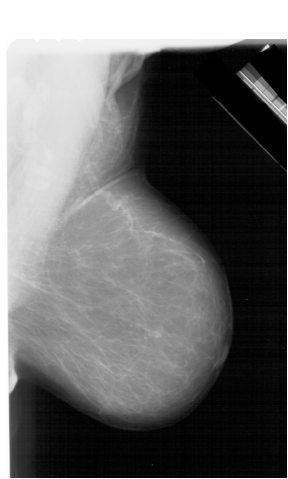

Reference (left)

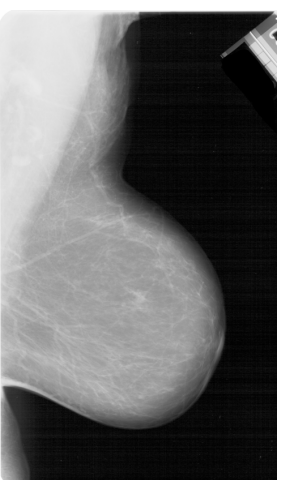

Target (right)

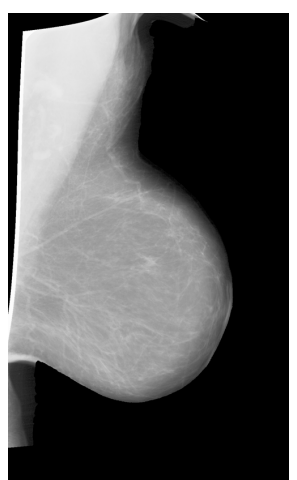

rpm

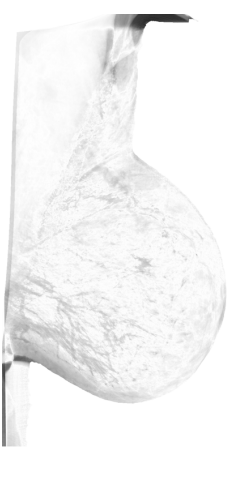

Difference

Fig. 2. Example of registration results using the rpm approach

\subsection{Quantitative Evaluation}

A total of 128 mammographic images obtained from the DDSM mammographic database [10] are used as initial evaluation. These include 64 different patients with left and right MLO images where a malignant mass has been detected and annotated as ground truth. The difference image (after histogram matching) is computed from the registered images for each patient. In the ideal case of a perfect registration, this image is likely to highlight the suspicious region. The idea is that results from the difference image could be used for mass detection or at least to reduce the number of false positives in mass detection algorithms. An evaluation on the distance parameters of the proposed method is firstly given, and subsequently, a comparison with other approaches is performed.

Distance Function. As mentioned before, different distance measures can be used for computing the cost matrix. Various experiments have been carried out in order to assess the benefits of each distance measure and its relative importance. Distance measures evaluated are Euclidean distance $(E)$ and shape contexts $(S C)$ each one weighted with the intensity information provided by the 
normalised cross-correlation $(N C C)$. The results of four different experiments $(A, B, C, D)$ are shown in Fig. 3. Each experiment evaluates the weighting factor $(\alpha)$ between the two distance measures used. In experiments $\mathrm{A}$ and $\mathrm{B}$ the measures evaluated are $E$ and $N C C$ for boundary $(A)$ and for boundary and internal points $(B)$. Experiments $\mathrm{C}$ and $\mathrm{D}$ use $S C$ and $N C C$ again for boundary $(C)$ and internal points $(D)$. A different curve is shown for each experiment showing the goodness of the registration as a function of the weighted distance measures. Goodness of the registration is computed using the mean value of a similarity measure (i.e. mutual information) for all 64 patients. As expected, and corroborated by visual inspection of the registered images, the worst results are obtained using only breast boundary points (experiments $A$ and $C$ ). For experiments using internal points $(B$ and $D)$, boundary matches are initially found using the best results of the experiments $A$ and $C$. Therefore, better registration results should be obtained assuming that those internal points are correctly detected. This is corroborated by the experiments, where $B$ and $D$ outperform the best results of the experiments using boundary points alone. The experiment also shows that Euclidean distance alone provides good registration results while shape contexts needs additional grey level information to reach similar levels.

Comparing with other approaches. This section shows the initial results of the proposed method compared to other approaches. The approaches evaluated are global image registration using affine transformation maximising a mutual information measure (miat), image registration using our previously presented approach based on point matching and thin-plate splines 4] (linreg and linreg $B B$ ) and the proposed method (rpm and rpmBB). Here, $B B$ denotes that the same method is used but only taking the breast boundary into account. The miat implementation is similar to one of the evaluated methods presented in 3 . Fig. 4a shows evaluation results in terms of box plots computed from similarity

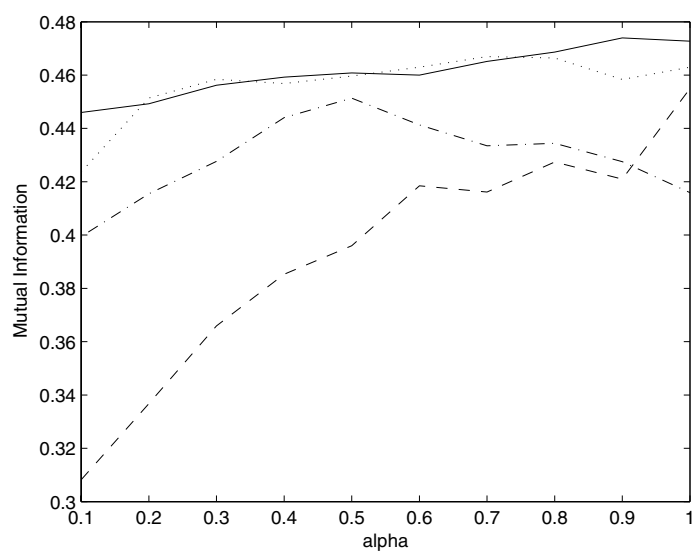

Fig. 3. Evaluation results of the relative importance of the distance measures in the final registration result: Experiments $A$ (dashed), $B$ (solid) and $C$ (dot dashed) and $D$ (dotted) 
measures (mutual information) between the reference image (left breast) and the registered image (right breast) using the different approaches. From the plots, we can conclude that the proposed method obtains a similar accuracy to the linreg method with a slightly higher mean value and more robust and stable results (note the outlier in the linreg method). Results also show, as reported by various authors, that although using breast boundary information alone obtains good results, accuracy and robustness is increased when information from the internal breast structure is included. Additional evaluation results are shown in Fig. \b, which shows ROC curves obtained from the difference image compared to the annotation ground truth provided by radiologists. The ROC curve is build by measuring the true positive and false positive fraction as a function of a threshold of the difference image compared to manual segmentations provided by a radiologist. In this case, although curves get close for the cases of linreg, linreg $B B$ and rpm, analysing the Area Under the Curve $(A U C)$ value our proposal has a slightly worst results.

In summary, the proposed method obtains better results compared to the miat method which is in contrast with the results published in [3. This will need further investigation but could be due to particular implementations or to pre-processing steps (i.e. pectoral muscle suppression). Compared to the linreg method, similar but more robust results are obtained. Both approaches share common methodologies which explains the similarity of the results. A comparison with other recently published approaches 5 can not be directly stated from this work but additional evaluation procedures will be proposed and included in the future work.

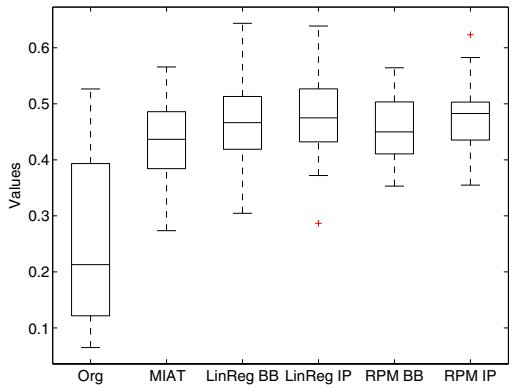

(a)

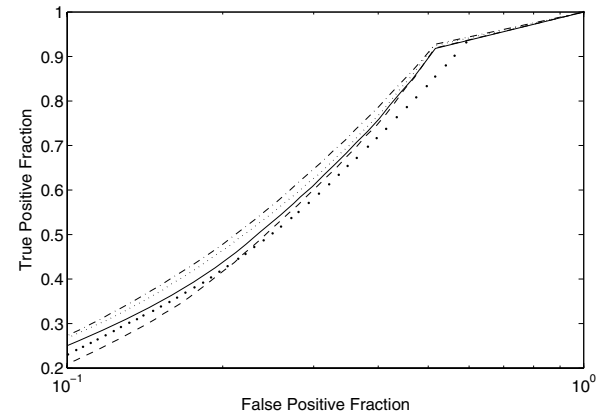

(b)

Fig. 4. (a) Box plots and (b) ROC curves for different registration methods: miat (bold dotted), linregBB (dotted), linreg (dash dotted), rpmBB (dashed) and rpm (solid). $A U C$ values for each method are $0.714,0.739,0.747,0.722$ and 0.730 , respectively.

\section{Conclusions}

A novel registration algorithm has been presented based on the application of robust point matching concepts. Quantitative and qualitative results have been presented that show the validity of our approach. Although initial results are 
presented, a comparison with other approaches has been provided, showing reduced error rates for the developed method. Future work will focus on extending the number of cases including temporal studies from our local database. Additional evaluation in terms of landmark error measures will be presented with the aim to obtain better comparison with other approaches.

\section{References}

1. Sallam, M., Bowyer, K.: Registration and difference analysis of corresponding mammogram images. Medical Image Analysis 3(2) (1999) 103-118

2. Karssemeijer, N., Brake, G.T.: Combining single view features and asymmetry for detection of mass lesions. In Karssemeijer, N., Thijssen, M., Hendriks, J., van Erning, L., eds.: $4^{\text {th }}$ International Workshop on Digital Mammography, Kluwer Academic (1998) 95-102

3. van Engeland, S., Snoeren, P., Hendriks, J., Karssemeijer, N.: A comparison of methods for mammogram registration. IEEE Transactions on Medical Imaging 22(11) (2003) 1436-1444

4. Marti, R., Zwiggelaar, R., Rubin, C.: Automatic point correspondence and registration based on linear structures. International Journal of Pattern Recognition and Artificial Intelligence 16(3) (2002) 331-340

5. Marias, K., Behrenbruch, C.P., Parbhoo, S., Seifalian, A., Brady, M.: A registration framework for the comparison of mammogram sequences. IEEE Transactions on Medical Imaging 24(6) (2005) 782-790

6. Besl, P.J., McKay, N.D.: A method for registration of 3-D shapes. IEEE Transactions on Pattern Analysis and Machine Intelligence 14(2) (1992) 239-256

7. Belongie, S., Malik, J., Puzicha, J.: Shape matching and object recognition using shape contexts. IEEE Transactions on Pattern Analysis and Machine Intelligence 24(4) (2002) 509-522

8. Chui, H., Rangarajan, A.: A new point matching algorithm for non-rigid registration. Computer Vision and Image Understanding 89(2-3) (2003) 114-141

9. Rohr, K., Stiehl, H.S., Sprengel, R., Buzug, T.M., Weese, J., Kuhn, M.H.: Landmark-based elastic registration using approximating thin-plate splines. IEEE Transactions on Medical Imaging 20(6) (2001) 526-534

10. Heath, M., Bowyer, K., Kopans, D., Moore, R., Kegelmeyer, P.: The digital database for screening mammography. In: International Workshop on Digital Mammography. (2000) 